\title{
Performance Improvement of Spoke-shaped Interior Permanent Magnet Magnetic Gear
}

\author{
Y. Mizuana, K. Nakamura, Y. Suzuki*, Y. Oishi*, Y. Tachiya*, and K. Kuritani* \\ Graduate School of Engineering, Tohoku University, 6-6-11 Aoba Aramaki Aoba-ku, Sendai, Miyagi 980-8579, Japan \\ *Prospine Co., Ltd., 117 Shinsengarida, Matsuyamatsugihashi, Osaki, Miyagi 987-1305, Japan
}

\begin{abstract}
Magnetic gears have several advantages such as having low acoustic noise and being maintenance-free because there is no mechanical contact. Among these gears, the flux-modulated type has a higher torque density and efficiency than the other types. Therefore, it is expected to be put into practical use. In previous papers, a spoke-shaped interior permanent magnet (IPM) rotor structure was proposed in order to reduce the eddy current loss in magnets. This paper presents three methods for improving the performance of the spoke-shaped IPM magnetic gear. First, the position of the magnetic bridges is changed from the air gap side to the back yoke side to increase the effective magnetic flux. Second, amorphous alloy is used for the rotor core and pole pieces in order to reduce iron loss. Third, bonded Nd-Fe-B magnets for canceling the leakage flux are arranged to sandwich the inner rotor from both sides. As a result of prototype tests, the measured maximum torque of the proposed spoke-shaped IPM magnetic gear was $44 \%$ higher than that of the conventional one. In addition, the measured maximum efficiency reached $99.85 \%$ at $100 \mathrm{rpm}$.
\end{abstract}

Key words: magnetic gear, interior permanent magnet (IPM), cancel magnet, amorphous alloy, magnetic bridge

\section{Introduction}

Mechanical gears are one of the most important mechanical elements. Thus, they are widely used in various fields such as industry, transportation, and power generation. However, the mechanical gears have several problems including acoustic noise and vibration due to mechanical contact. Furthermore, they require lubricant for cooling and wear reduction, and periodic maintenance.

On the other hand, magnetic gears change speed and transmit torque without any mechanical contacts. Therefore, they have several advantages of low acoustic noise, low vibration, and maintenance-free compared to mechanical gears. In previous researches ${ }^{1)-3)}$, various types of magnetic gears have been introduced. Among them, a flux-modulated type magnetic gear ${ }^{4)-5)}$ has attracted attention recently.

Fig. 1 shows a basic structure of flux-modulated type magnetic gear. It consists of the inner and outer rotors and pole pieces placed between the both rotors. This magnetic gear works as a gear when the magnet flux is modulated by pole pieces. Since all the magnets of the inner and outer rotors contribute to torque transmission, a flux-modulated type magnetic gear has higher torque density and efficiency than the other types ${ }^{6)-7)}$.

For the practical use of a flux-modulated type magnetic gear, further torque improvement and loss reduction are necessary. In previous papers ${ }^{8)-9)}$, a spoke-shaped interior permanent magnet (IPM) magnetic gear was proposed for the reduction of eddy current loss in magnets caused by asynchronous harmonic fluxes.

This paper presents three ideas to improve the performance of the spoke-shaped IPM magnetic gear.
First, some problems with conventional spoke-shaped IPM magnetic gear are pointed out. Next, solutions to the above problems are proposed and evaluated by using JMAG-Designer ver. 19.1, which is general-purpose finite element method (FEM) software. Finally, in order to prove the validity, prototype tests are demonstrated.

\section{Performance improvement of the spoke-shaped IPM magnetic gear}

2.1 Problems of conventional spoke-shaped IPM magnetic gear

Fig. 2 shows specifications of conventional spoke-shaped IPM magnetic gear. The diameter and axial length are $150 \mathrm{~mm}$ and $25 \mathrm{~mm}$, respectively. The numbers of pole-pairs of the inner and outer rotor are 3 and 31 , respectively. Hence, the gear ratio is 10.33 , which is given by the ratio of the inner and outer pole-pairs ${ }^{4)}$. The number of pole pieces is 34 given by the sum of the numbers of the inner and outer pole-pairs ${ }^{4)}$. The inner and outer magnets are embedded in the inner and outer rotor core as a spoke shape. Both rotors have $1 \mathrm{~mm}$-wide magnetic bridges on the air gap side. Non-magnetic stainless steel is used

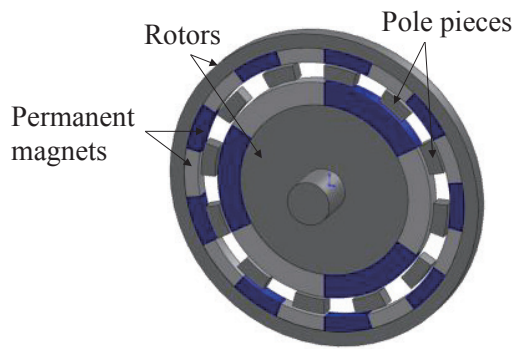

Fig. 1 Basic structure of flux-modulated type magnetic gear. 


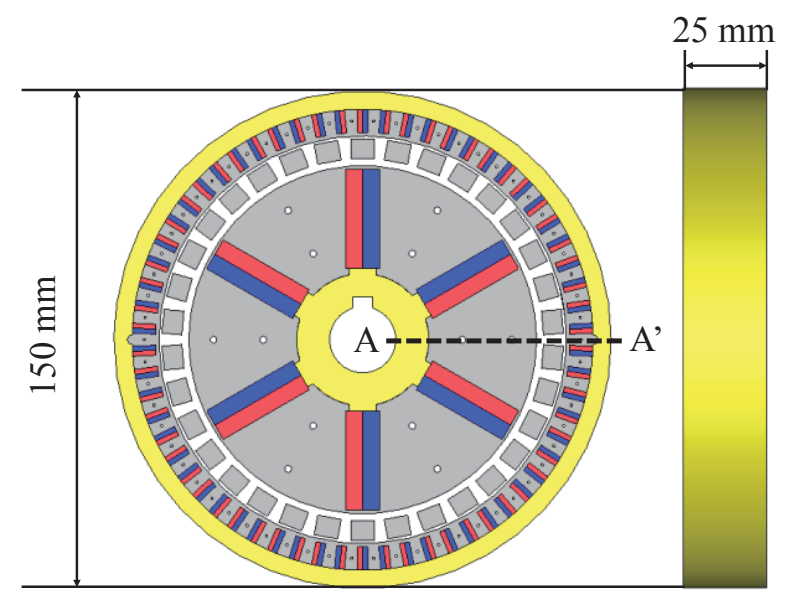

\begin{tabular}{ll}
\hline \hline Bridge position & Air gap side \\
Bridge width & $1 \mathrm{~mm}$ \\
Cancel magnets & Not included \\
& $2 \mathrm{~mm}$ (inner) \\
Air gap & $1 \mathrm{~mm}$ (outer) \\
Inner pole-pairs & 3 \\
Outer pole-pairs & 31 \\
Number of pole pieces & 34 \\
Gear ratio & 10.33 \\
Core material & $35 \mathrm{~A} 250$ \\
Pole-piece material & SMC \\
Magnet material & Sintered Nd-Fe-B \\
\hline \hline
\end{tabular}

Fig. 2 Specifications of conventional spoke-shaped IPM magnetic gear.

for the rotor back yoke to prevent a short circuit of the PM flux. Core material of the both rotors is non-oriented silicon steel with a thickness of $0.35 \mathrm{~mm}$ (35A250), while that of pole-pieces is soft magnetic composite (SMC).

Fig. 3 (a) illustrates a vector diagram of magnetic flux density of conventional spoke-shaped IPM magnetic gear. From the figure, it is understood that a part of the PM flux flows through the magnetic bridges, which leads to reduction of effective PM flux.

Fig. 3 (b) indicates losses of conventional spoke-shaped IPM magnetic gear calculated by 3D-FEM. As shown in the figure, the eddy current loss in the magnets is very small due to the spoke-shaped IPM rotor structure, while the iron loss is large. For improvement of efficiency, the reduction of iron loss is critical.

Fig. 3 (c) shows leakage flux of conventional spoke-shaped IPM magnetic gear (A-A' cross section in Fig. 2). From the figure, it is clear that the axial leakage flux is more severe in the inner rotor than the outer rotor because the larger size magnets are arranged facing each other. It is concerned that the axial leakage flux induces eddy current in the gear housing and decreases the efficiency.

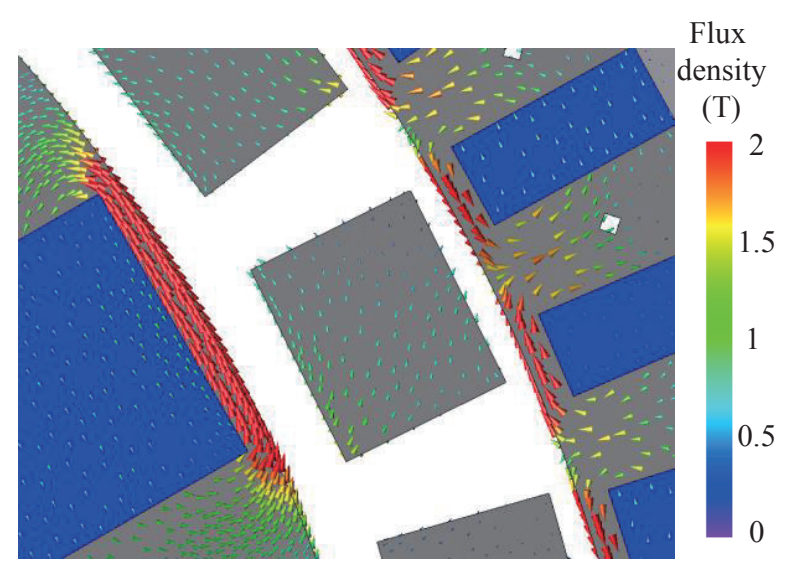

(a) Vector diagram of magnetic flux density of conventional spoke-shaped IPM magnetic gear.

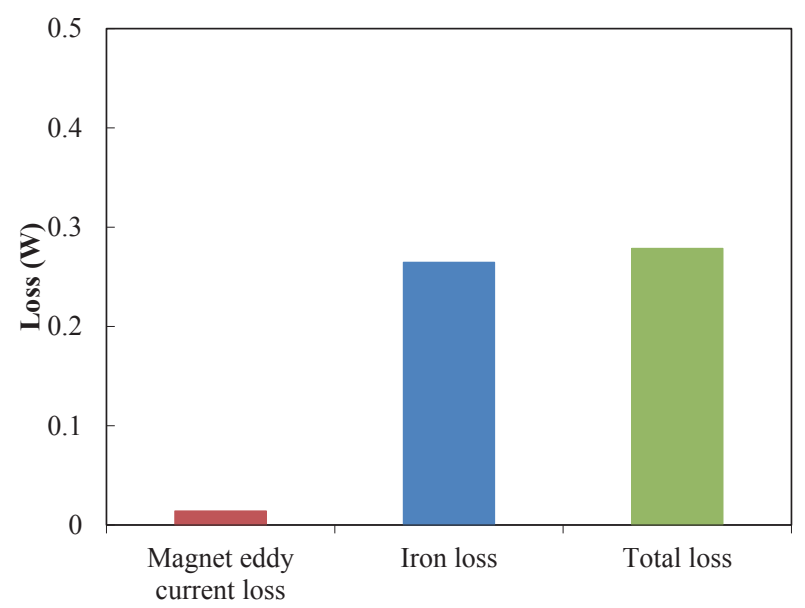

(b) Losses of conventional spoke-shaped IPM magnetic gear calculated by 3D-FEM.

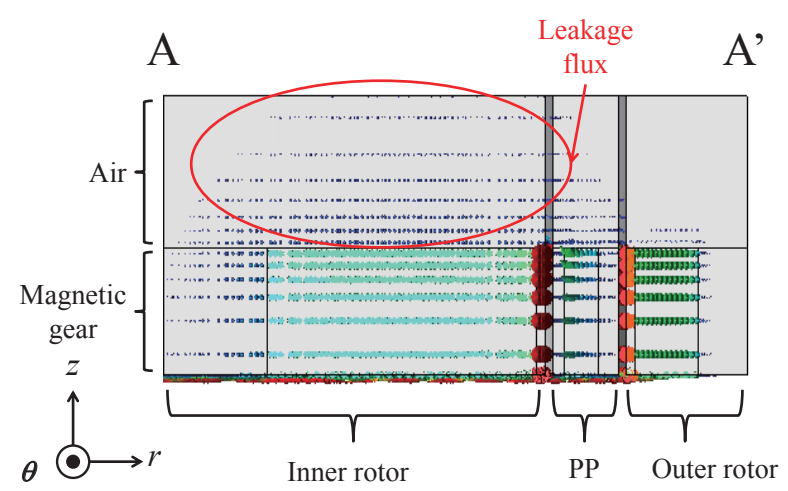

(c) Leakage flux distribution of conventional spoke-shaped IPM magnetic gear (A-A' cross section in Fig. 2).

Fig. 3 Problems with conventional spoke-shaped IPM magnetic gear.

\subsection{Methods for performance improvement}

Fig. 4 shows specifications of proposed spoke-shaped IPM magnetic gear. For the performance improvement, three ideas are employed for the proposed magnetic gear. 


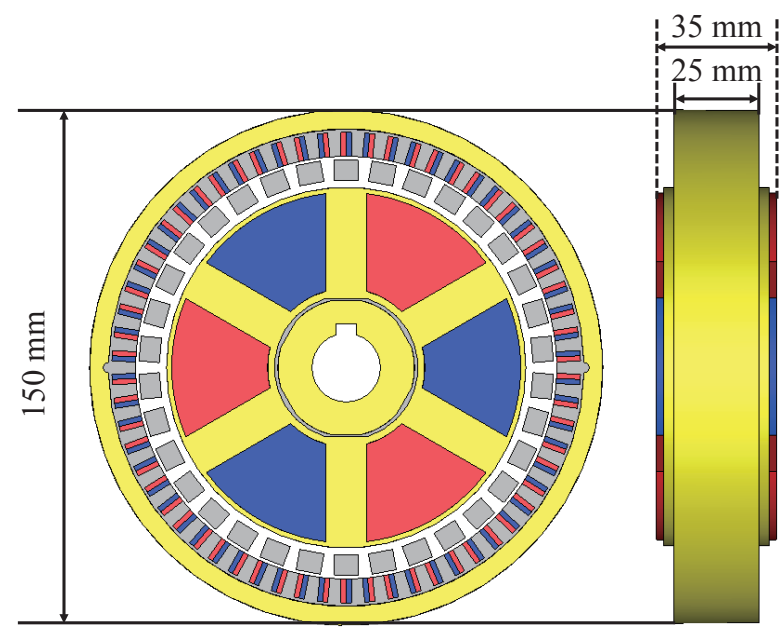

\begin{tabular}{ll}
\hline \hline Bridge position & Back yoke side \\
Bridge widths & $2 \mathrm{~mm}$ (inner) \\
& $1 \mathrm{~mm}$ (outer) \\
Cancel magnets & Included (5mm) \\
Air gap & $1 \mathrm{~mm}$ (inner) \\
Inner pole-pairs & 3 \\
Outer pole-pairs & 31 \\
Number of pole pieces & 34 \\
Gear ratio & 10.33 \\
Core material & Amorphous alloy \\
Pole-piece material & Amorphous alloy \\
Magnet material & Sintered Nd-Fe-B \\
\hline
\end{tabular}

Fig. 4 Specifications of proposed spoke-shaped IPM magnetic gear.

First, for increasing the effective PM flux, the position of the magnetic bridges is changed from the air gap side to the back yoke side as shown in Fig. 5. Fig. 6 indicates magnetic flux density waveforms in outer air gap. From the figure, it is understood that the magnetic flux in outer air gap is increased by arranging the magnetic bridges on the back yoke side.

Second, in order to reduce iron loss, amorphous alloy is used for the inner and outer rotor core, and pole pieces. Fig. 7 shows comparison of total loss when the inner rotor rotates at $300 \mathrm{rpm}$. As shown in the figure, it is revealed that the total loss of the proposed magnetic gear can be significantly reduced by employing amorphous alloy for pole pieces.

Third, bonded $\mathrm{Nd}-\mathrm{Fe}-\mathrm{B}$ magnets for canceling the axial leakage flux are arranged to sandwich the inner rotor from both sides as shown in Fig. 8. These cancel magnets are magnetized in the opposite direction to the axial leakage flux and fixed by the stainless steel frame. The thickness of the cancel magnets was optimized to 5 $\mathrm{mm}$ so that the average axial leakage flux on surface of cancel magnets is zero as shown in Fig. 9.

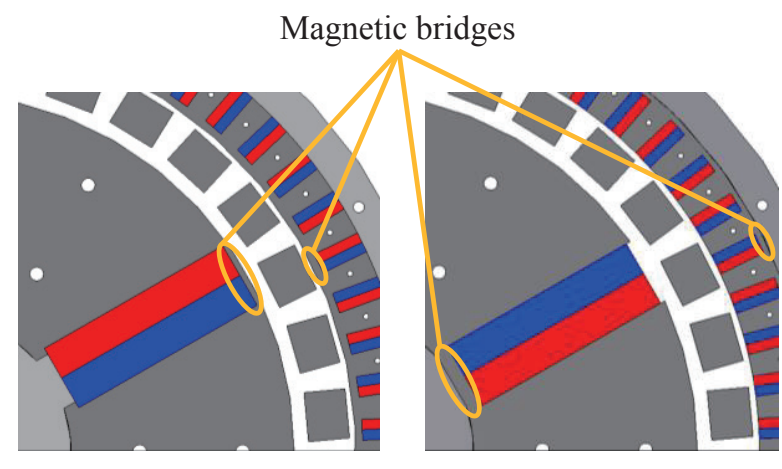

Conventional (air gap side) Proposed (back yoke side)

Fig. 5 Position of magnetic bridges of conventional and proposed spoke-shaped IPM magnetic gears.

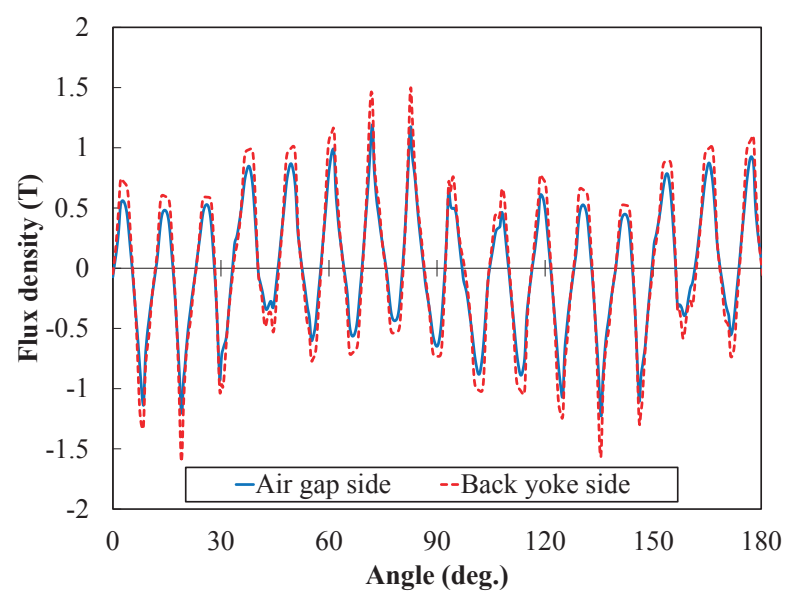

Fig. 6 Magnetic flux density waveforms in outer air gap.

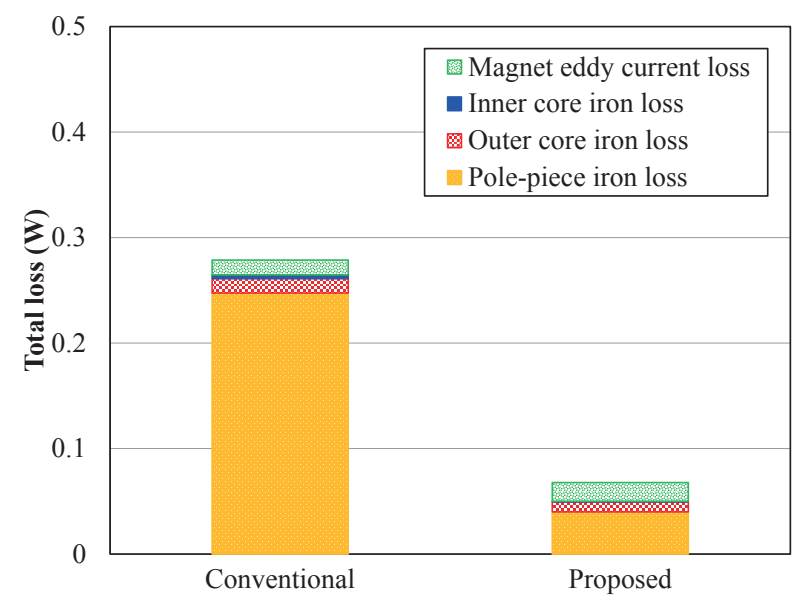

Fig. 7 Comparison of calculated total loss when inner rotational speed was $300 \mathrm{rpm}$.

Fig. 10 indicates the radial magnetic flux density waveforms in inner air gap with and without cancel magnets. From, the figure, it is understood that the magnetic flux in inner air gap is enhanced by the cancel magnets. Therefore, the cancel magnets are expected to contribute to the torque improvement in addition to canceling the axial leakage flux. 


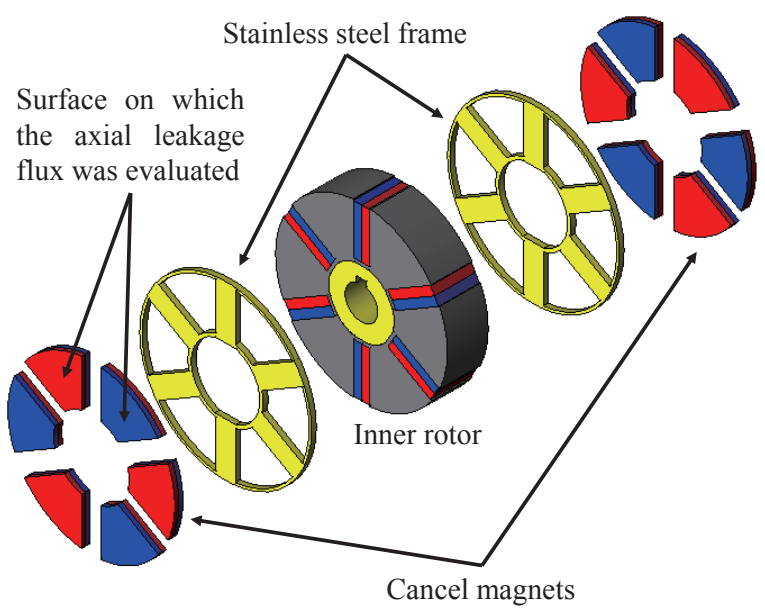

Fig. 8 Exploded view of inner rotor with cancel magnets.

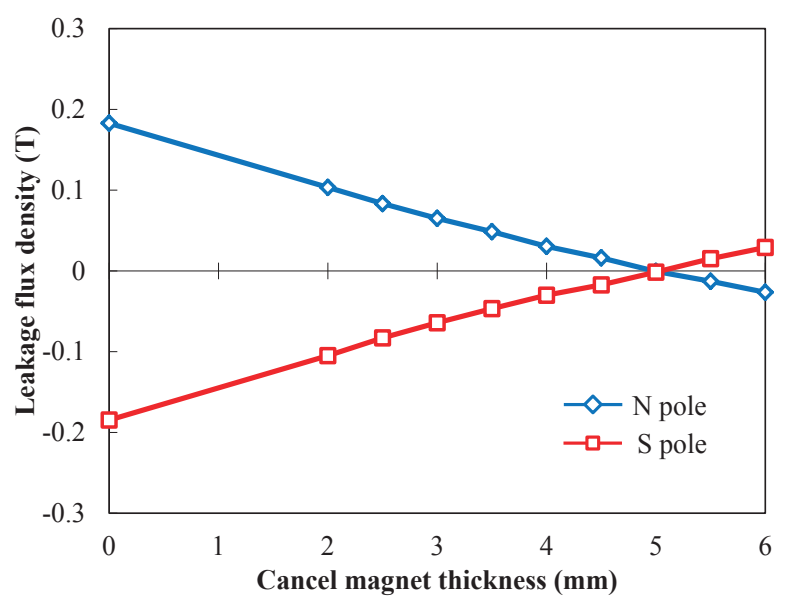

Fig. 9 Calculated average axial leakage flux density on surface of cancel magnets.

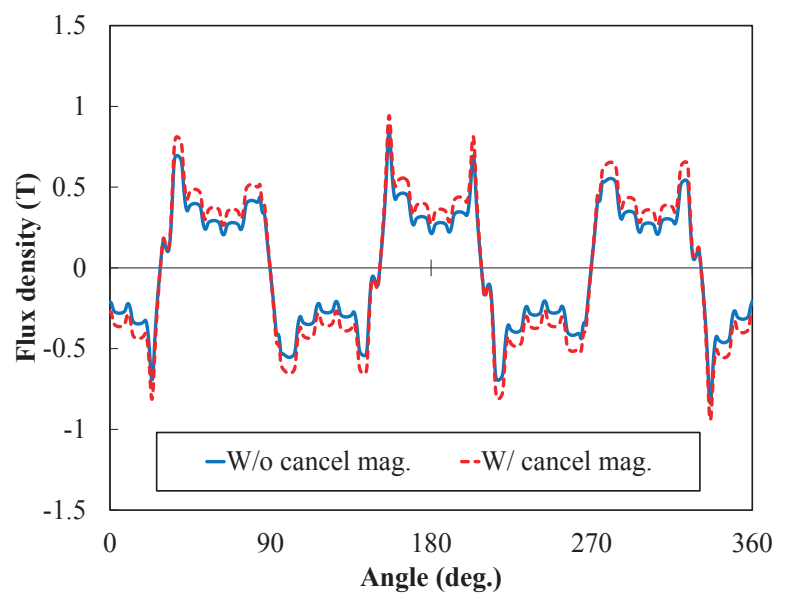

Fig. 10 Calculated radial magnetic flux density waveforms in inner air gap with and without cancel magnets.

Fig. 11 shows comparison of the maximum torque when a rotational speed of the inner rotor is $300 \mathrm{rpm}$. The figure reveals that the maximum torque of the proposed magnetic gear is larger than that of the conventional one, and that amorphous alloy also contributes to the torque improvement despite low saturation magnetic flux density.

To clarify the above reason, Fig. 12 (a) and Fig. 12 (b) indicate contour diagrams of magnetic flux density when the rotor core materials are 35A250 and amorphous alloy, respectively. Pole piece material is SMC in both cases. As shown in the figure, it is clear that the magnetic bridges are magnetically saturated at lower magnetic flux density when amorphous alloy is used for the rotor core, which increases the effective PM flux, and results in the improvement of torque.

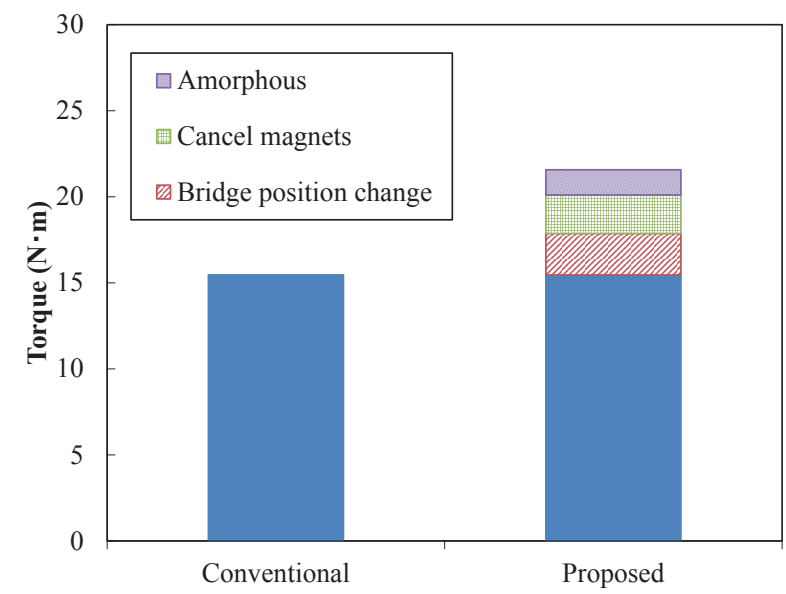

Fig. 11 Comparison of calculated maximum torque.

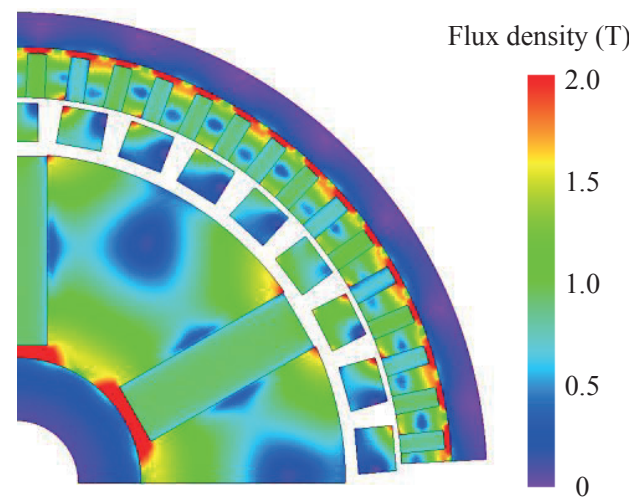

(a) Non-oriented silicon steel (35A250)

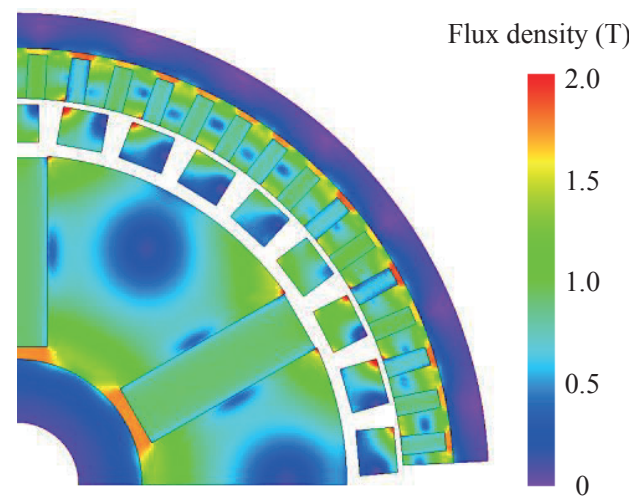

(b) Amorphous alloy

Fig. 12 Contour diagrams of magnetic flux density. 


\section{Prototype tests of spoke-shaped IPM magnetic gear}

Based on the simulation results of the previous chapter, the proposed spoke-shaped IPM magnetic gear was prototyped and its performance was compared with that of the conventional one.

First, the effect of the cancel magnets is verified. Fig. 13 shows the experimental setup for measuring leakage flux. The axial leakage flux is measured with a Gauss meter at the five points shown in Fig. 14.

Table 1 indicates the measured axial leakage flux with and without the cancel magnets. From the table, it is proved that the axial leakage flux can be remarkably reduced by the cancel magnets.

Fig. 15 shows appearance of prototyped spoke-shaped IPM magnetic gear. As shown in the figure, it has an observation hole for measuring the leakage flux and the temperature inside the prototype gear. The gear housing is made of fiber reinforced plastic (FRP) in order to prevent generation of losses caused by stray electromagnetic field.

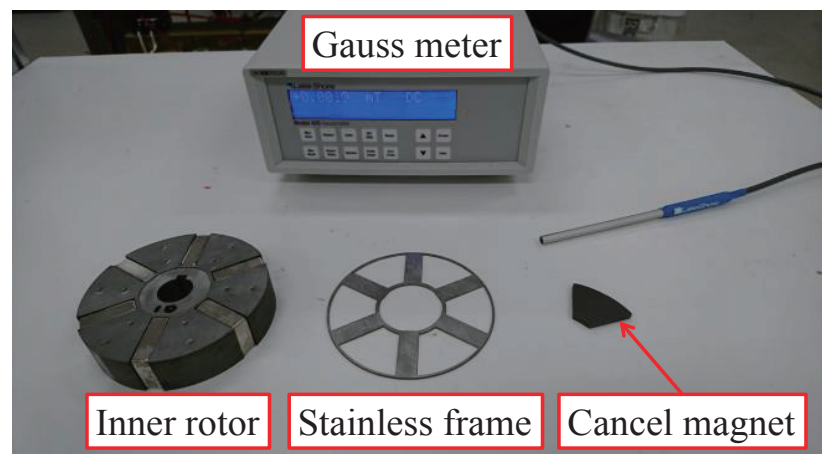

Fig. 13 Experimental setup for measuring leakage flux.

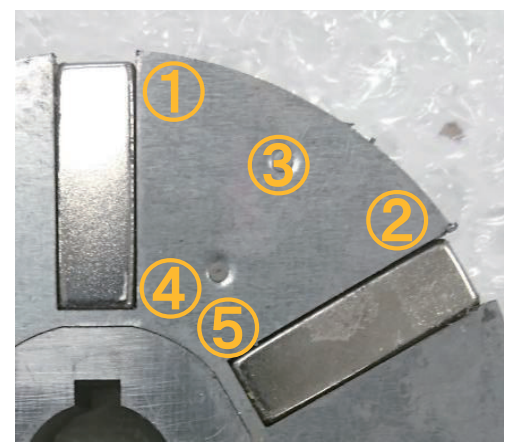

Fig. 14 Measured points.

Table 1 Measured leakage flux density.

\begin{tabular}{ccc}
\hline \hline $\begin{array}{c}\text { Measured } \\
\text { point }\end{array}$ & $\begin{array}{c}\text { W/o cancel } \\
\text { magnets }\end{array}$ & $\begin{array}{c}\text { W/ cancel } \\
\text { magnets }\end{array}$ \\
\hline 1 & $0.229 \mathrm{~T}$ & $0.005 \mathrm{~T}$ \\
2 & $0.262 \mathrm{~T}$ & $0.001 \mathrm{~T}$ \\
3 & $0.144 \mathrm{~T}$ & $0.006 \mathrm{~T}$ \\
4 & $0.243 \mathrm{~T}$ & $0.012 \mathrm{~T}$ \\
5 & $0.258 \mathrm{~T}$ & $0.012 \mathrm{~T}$ \\
\hline \hline
\end{tabular}

Fig. 16 indicates the experimental system for prototyped magnetic gear. In this system, the prototype magnetic gear operates as a step-up gear. The rotational speed of the outer rotor is regulated at an arbitrary speed by the servomotor. The load torque is controlled by the hysteresis brake.

Fig. 17 shows comparison of the torque behavior at a constant speed of the inner rotor of $300 \mathrm{rpm}$. As shown in the figure, the load torque is increased stepwise until the magnetic gear is stepped out. The figure reveals that the measured maximum torque of the proposed spoke-shaped IPM magnetic gear is $44 \%$ higher than that of the conventional one. On the other hand, the measured torque is about $10 \%$ lower than the calculated one. This is because magnetic flux leaks more than expected by FEM. In IPM magnetic gear, the same poles of the inner and outer magnets face each other so that magnetic flux flows toward the pole pieces. However, not all of the magnetic flux flows toward the pole piece, and some flux leaks in various directions. In addition, flat structure shown in Fig. 2 contributes to increasing the leakage flux. For these reasons, effective magnetic flux decreases, and the measured torque is smaller.

Fig. 18 shows comparison of the measured efficiency at a constant speed of the inner rotor of 300 and $100 \mathrm{rpm}$, respectively. The figure demonstrates that the efficiency of the proposed spoke-shaped IPM magnetic gear is remarkably improved, and the measured maximum efficiency of the proposed spoke-shaped IPM magnetic gear reaches $99.85 \%$ at an inner rotor speed of $100 \mathrm{rpm}$.
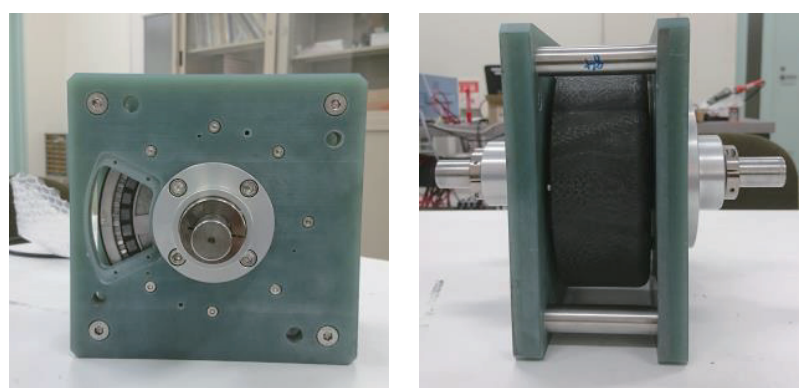

Fig. 15 Appearance of prototyped spoke-shaped IPM magnetic gear.

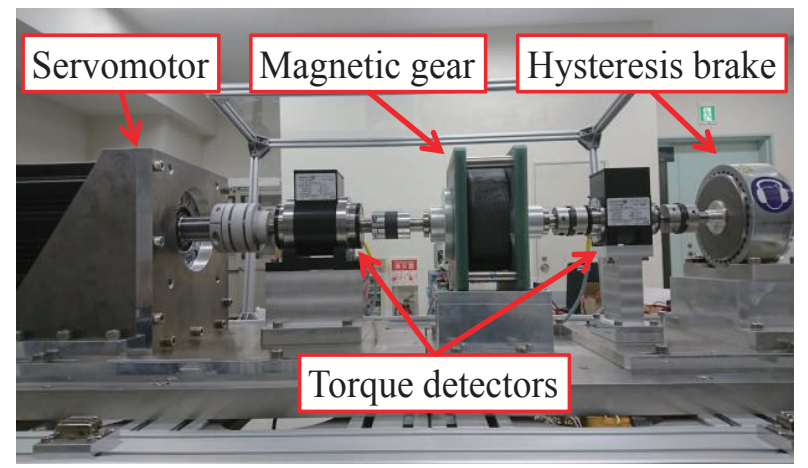

Fig. 16 Experimental system for prototyped magnetic gear. 


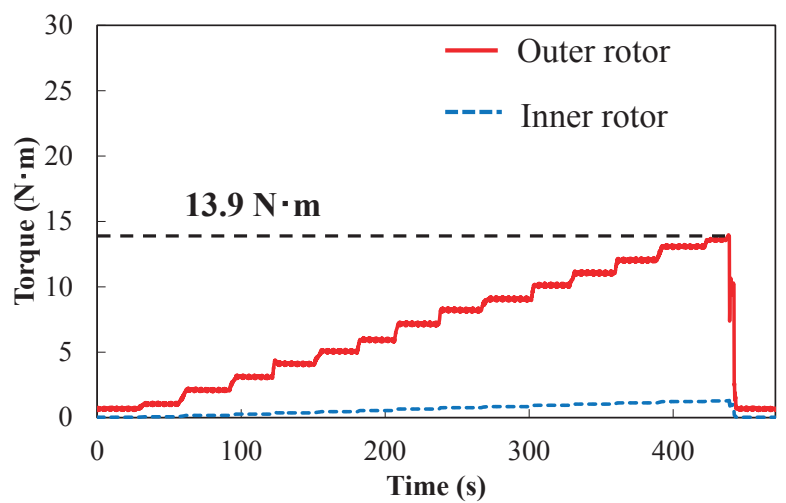

(a) Conventional spoke-shaped IPM magnetic gear.

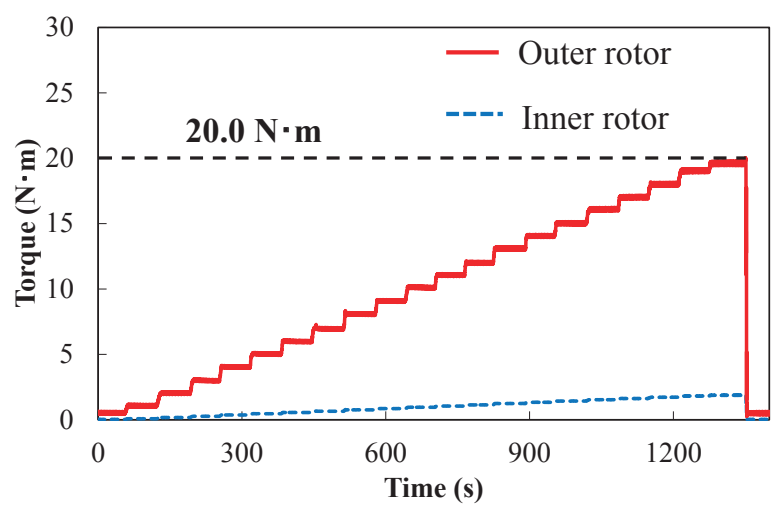

(b) Proposed spoke-shaped IPM magnetic gear.

Fig. 17 Measured torque behavior of conventional and proposed spoke-shaped IPM magnetic gears.

\section{Conclusion}

This paper presented three methods for improving the performance of the spoke-shaped IPM magnetic gear. First, a position of magnetic bridges is changed from the air gap side to the back yoke side. Second, amorphous alloy is employed for rotor cores and pole pieces. Third, bonded Nd-Fe-B magnets are arranged to sandwich the inner rotor from both sides in order to cancel the axial leakage flux.

From the simulation results, it was revealed that all three methods are effective in the performance improvement of the spoke-shaped IPM magnetic gear.

In the prototype tests, the axial leakage flux was reduced by more than $95 \%$ by using the cancel magnets. Furthermore, it was demonstrated that the proposed spoke-shaped IPM magnetic gear has 44\% larger torque than the conventional one, and the measured maximum efficiency reaches $99.85 \%$ at an inner rotational speed of $100 \mathrm{rpm}$.

Acknowledgements This work was supported in part by the WISE Program for AI Electronics, Tohoku University.

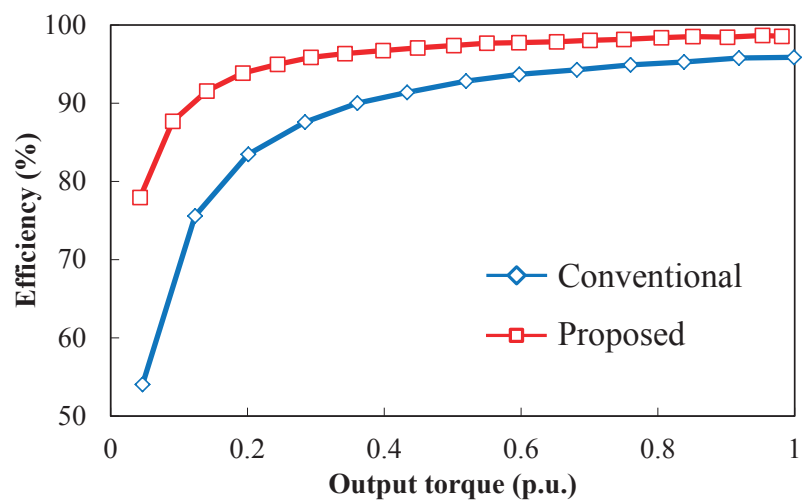

(a) Inner rotational speed : $300 \mathrm{rpm}$

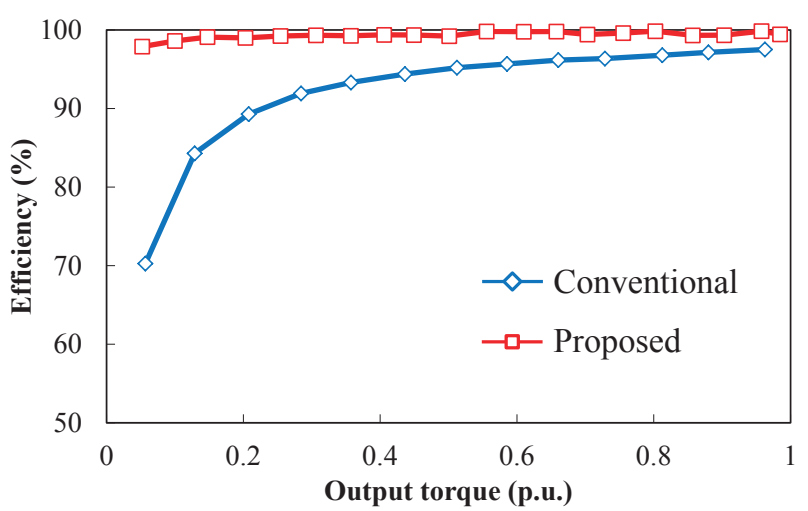

(b) Inner rotational speed : $100 \mathrm{rpm}$

Fig. 18 Measured efficiency of conventional and proposed spoke-shaped IPM magnetic gears.

\section{References}

1) T. B. Martin, Jr.: U.S. Patent, 3, 378, 710 (1968).

2) D. E. Hesmondhalgh and D. Tipping: IEE Proc. B, Elect. Power Appl., 127, 129 (1980).

3) K. Tsurumoto and S. Kikushi: IEEE Trans. Magn., 23, 3622 (1987).

4) K. Atallah and D. Howe: IEEE Trans. Magn., 37, 2844 (2001).

5) F. T. Jørgensen, P. O. Rasmussen, and T. O. Andersen: Summer Seminar on Nordic Network for Multi Disciplinary Optimized Electric Drives (2003)

6) T. Ikeda, K. Nakamura, and O. Ichinokura: J. Magn. Soc. Jpn., 33, 130 (2009) (in Japanese).

7) M. Fukuoka, K. Nakamura, and O. Ichinokura: IEEJ Trans. A, 134, 416 (2014) (in Japanese).

8) T. Ikeda, K. Nakamura, and O. Ichinokura: J. Magn. Soc. Jpn., 34, 380 (2010) (in Japanese).

9) Y. Mizuana, K. Nakamura, Y. Suzuki, Y. Oishi, Y. Tachiya, and K. Kuritani: International Journal of Applied Electromagnetics and Mechanics (2020).

Received Dec. 07, 2020; Accepted Jan. 26, 2021 